\title{
Prenatal is Still Neglected in Resource-Limited Areas despite the Use of Tests That Optimize the Diagnosis of Infectious Diseases in Brazil
}

\section{Galileu Barbosa Costa $^{1 *+}$, Raquel Gois Bastos ${ }^{2+}$, Ney Cristian Amaral Boa Sorte ${ }^{3,4}$, Antônio Conceição da Purificação ${ }^{3}$, Beneli Miranda dos Santos ${ }^{3}$, Bernardo Galvão-Castro ${ }^{4}$, Sara Nunez Vaz ${ }^{5}$, Lauro Juliano Marin ${ }^{2}$ and Sandra Rocha Gadelha ${ }^{2}$}

\begin{abstract}
${ }^{1}$ Núcleo de Epidemiologia e Bioestatística, Centro de Pesquisas Gonçalo Moniz, Fiocruz, Salvador, Brazil ${ }^{2}$ Laboratório de Farmacogenômica e Epidemiologia Molecular, Departamento de Ciências Biológicas, Universidade Estadual de Santa Cruz, Ilhéus, Bahia, Brazil

${ }^{3}$ Associação de Pais e Amigos dos Excepcionais (APAE), Salvador, Bahia, Brazil

${ }^{4}$ Escola Baiana de Medicina e Saúde Pública, Fundação Bahiana para Desenvolvimento das Ciências, Salvador, Bahia, Brazil

${ }^{5}$ Centro de Pesquisa Gonçalo Moniz, Fundação Oswaldo Cruz (FIOCRUZ), Salvador, Bahia, Brazil
\end{abstract}

*Corresponding author: Galileu Barbosa Costa, Núcleo de Epidemiologia e Bioestatística, Centro de Pesquisas Gonçalo Moniz, Fiocruz, Rua Waldemar Falcão, 121, Candeal, Salvador, Bahia, Brazil, 40296-710, Tel: +5573988112802;

Sandra Rocha Gadelha, Universidade Estadual de Santa Cruz, Departamento de Ciências Biológicas, Campus Soane Nazaré de Andrade, Rodovia Jorge Amado, km 16, Salobrinho, Ilhéus, Bahia, Brazil, 45662-900, Tel: +557388384075

\begin{abstract}
Vertically transmitted infections are caused by a diversity of pathogenic microorganisms, and pregnant women are routinely screened to evaluate the risks and reduce the burden of general disorders in their unborn. We assessed the seroprevalence of syphilis, toxoplasmosis, hepatitis B and C, HIV and Human T lymphotropic virus (HTLV) in pregnant women using public healthcare services in two main cities in South region of Bahia State, Brazil. Blood samples were collected using filter paper cards from 943 pregnant women aged between 12 and 45 years (average age of $24.3 \mathrm{yr}$ ). The average of pregnancies was 2.15 , and most women reported they had never experienced abortion. ELISA assays were applied to detect antibodies against HBV, HCV, HIV, HTLV, syphilis, and toxoplasmosis. The prevalence rates of $\operatorname{lgG}$ antibodies found were $0.2 \%$ for $\mathrm{HBV}, 0.1 \%$ for $\mathrm{HCV}$, $0.5 \%$ for HIV, $1.4 \%$ for HTLV, $2.8 \%$ for T. pallidum, and $1.5 \%$ for $T$. gondii. Only $47.5 \%$ of pregnant women returned to confirmatory tests. The rate of return for confirmatory test was low, even using tests that promote the diagnostic system. Our findings highlight how pre-natal care is still neglected, reinforcing the lack of awareness and the need for more incisive health education policies in the region.
\end{abstract}

\section{Keywords}

Pregnant women, Infectious diseases, Prenatal, Resource-limited areas, Public health

\section{Introduction}

Pregnant women, unborn fetuses, and neonates represent populations of high-risk individuals with increased susceptibility to infectious microbes that can pose significant health risks during pregnancy, depending on the pathogenesis and disease outcome [1-3]. The occurrence of infectious diseases during pregnancy is associated with spontaneous abortion, stillbirth, preterm delivery, and low birth weight [4,5], as well as persistent and long-lasting infections, even if not expressed at birth $[6,7]$.

Infections transmitted to the fetus results in severe birth defects such as microcephaly or even fetal death, and is usually inversely related to gestational age at ac- 


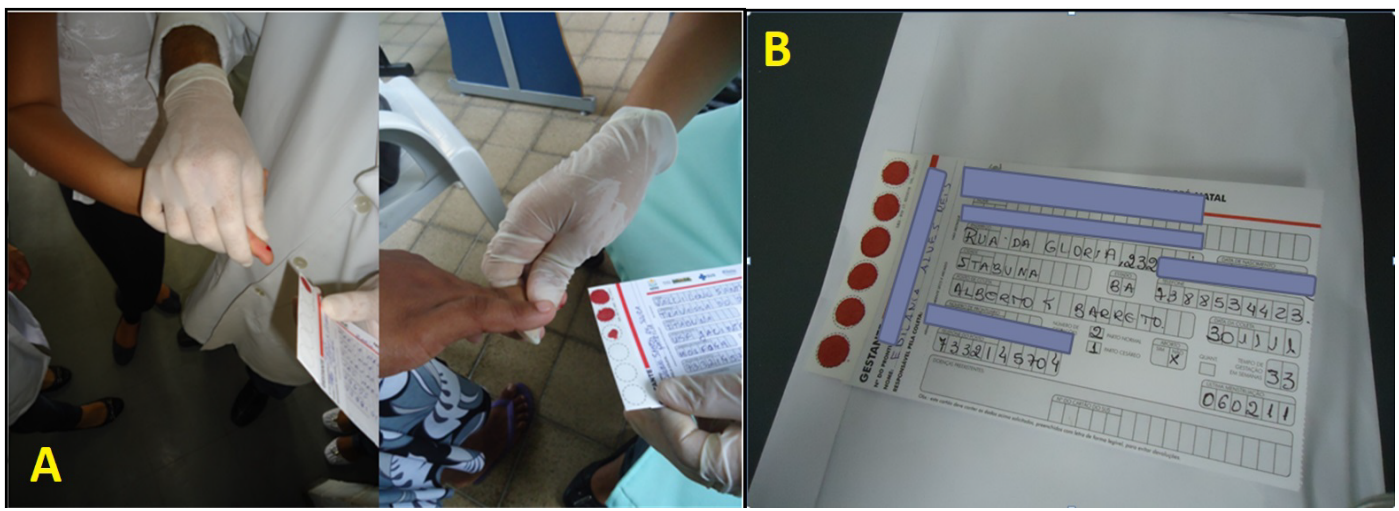

Figure 1: a) Blood sampling from pregnant women attended at Basic Healthcare Units from Itabuna and Ilhéus cities in the South State of Bahia. b) Filter paper correctly filled with blood samples and patient's basic information.

quisition [4,5]. Transmission can occur hematogenously from mother to the baby via placenta or as an ascending infection via the uterine cervix [8]. Postnatally transmission thought breast milk and other sources have also been documented as well [8].

The knowledge of the prevalence of infectious diseases in pregnant women populations has a great importance in the development of maternal and child healthcare policies $[9,10]$. The incidence of infectious diseases is variable in different populations and some studies have shown that these infections may occur in up to $10 \%$ of all live births. In Brazil, syphilis, toxoplasmosis, hepatitis $B$ and $C$, human immunodeficiency virus (HIV), and the human T-cells lymphotropic virus (HTLV) are some of the major infectious diseases found in pregnant women populations that may be transmitted to their fetus or newborn $[9,11,12]$.

Preventive programs have been implemented worldwide primarily aiming to eliminate the transmission of infectious diseases to children born from infected mothers [13]. However, limited therapeutic or preventive tools to protect the mother and the fetus during pandemics. Other factors that could also be associated to vulnerability to certain infectious diseases during pregnancy is a low income and education $[5,14]$.

Studies also report noncompliance with required examinations during pregnancy by many women. There are also records of pregnant women who use the service inappropriately, or even fail to participate in any of the prenatal care [15-17]. This lack of attendance could be explained mainly by the absence of an effective integration system between network services, and the complexity of operationalizing a more standardized prenatal care to facilitate the access of pregnant women to laboratory tests $[15,18]$.

In this study, we aimed to identify a variety of important infectious agents related to vertical transmission in a cohort of pregnant women from Southern region, State of Bahia, Brazil. In addition, our findings also bring an opportunity to discuss the difficult task of implement adequate diagnostics and the importance of health education in low-income populations.

\section{Material and Methods}

\section{Study population}

A cross-sectional study was carried out during November 2011-October 2012 with pregnant women attended at Basic Healthcare Units from two main cities in the South State of Bahia, Northeast region of Brazil.

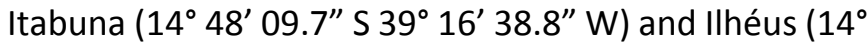
$\left.49^{\prime} 33.7^{\prime \prime} \mathrm{S} 39^{\circ} 02^{\prime} 03.7^{\prime \prime} \mathrm{W}\right)$. Data on age, number of pregnancies, number of abortions, and place of residence were collected and converted to variables to be tested for correlation with the screened pathogens.

\section{Samples collection and laboratory processing}

Drops of blood were collected by using sterile lancet in digital region in the first or second chirodactyl (Figures $1 \mathrm{~A}$ and $1 \mathrm{~B}$ ), after local asepsis with $70 \%$ ethanol $[19,20]$. The blood was soaked with three to four rounds of filter paper and dried at room temperature, protected from sunlight and from possible contaminants. After completely dried, the filter papers were bagged and kept at room temperature. The filter papers were sent to the Diagnostic and Research Center (CEDIP), APAE, Salvador, where they were processed and tested. Blood samples without anticoagulants were also collected, centrifuged and serum harvested and stored at $-20^{\circ} \mathrm{C}$ until tested.

\section{Detection of Hepatitis B and C, HIV, HTLV, Syphilis, and toxoplasmosis}

The samples were processed to detect anti-HIV 1+2, anti-HTLV-1 and 2, anti-VHC antibodies, anti T. pallidum (IgM and IgG), anti- T. gondii IgM antibodies and HBsAg on filter paper and plasma using the enzyme linked immunosorbent assay (ELISA). The immunological markers (antibodies) were obtained by an elution process, using a buffered solution containing protein, detergent, stabilizers and $0.1 \%$ sodium azide, and then subjected to ELI$\mathrm{SA}$, as recommended by the manufacturer. The following commercial kits on the material obtained on filter paper were used: IMUNOSCREEN HIV 1+2 - SS ${ }^{\odot}$, IMUN- 
OSCREEN HTLV I and II - SS ${ }^{\odot}$, IMUNOSCREEN ANTI-HCVSS ${ }^{\odot}$, IMUNOSCREEN TOXO IGM - SS ${ }^{\odot}$, IMUNOSCREEN SÍFILIS - SS ${ }^{\odot}$ and IMUNOSCREEN HBSAG - SS ${ }^{\odot}$, produced by MBIOLOG Diagnósticos LTD, Contagem, MG, Brazil.

\section{Confirmatory tests}

The active search system, in order to collect new samples to confirm the result obtained by the filter, was coordinated primarily by the APAE Salvador, through the specific sector, informing the Health Department of each city the pregnant woman results (positive screened cases). Since these women tend not to seek reference services frequently, when altered results were detected in the screening, it became necessary to seek these pregnant women in each Basic Health Unit, interfering with the service's natural flow. In Ilhéus, a collection point for weekly collections of these samples was set up. In Itabuna, some of these samples were collected in the existing laboratory at the reference center, and others, in their own health units. Reference services for the monitoring of pregnant women are: Women's Health Reference Center (CMAE) in Ilhéus and Reference Center Dr. Julio Brito (CRJB) in Itabuna. Of the pregnant women who answered the recall, two tubes of $4 \mathrm{ml}$ venous blood were collected, with one dry tube, and the other containing the anticoagulant Ethylenediamine tetraacetic acid (EDTA). After blood samples were collected, they were taken to the Pharmacogenomics Laboratory of Molecular Epidemiology of the UESC (LAFEM), where they were centrifuged at 2500 revolutions per minute (RPM) for five minutes to separate the serum and the cellular portion. The serum obtained from the dried tube was aliquoted into two wells of $2.0 \mathrm{ml}$, with one stored and the other sent to the APAE Salvador, where the confirmatory test was performed. The tube containing EDTA were also aliquoted into two microcentrifuge tubes for subsequent DNA extraction.

\section{Sequencing and subtyping of HIV and HTLV posi- tive samples}

Genomic DNA was extracted by using the QIAGEN QIAamp ${ }^{\circledR}$ DNA Blood Kit, according to the manufacturer's instructions. All DNA samples were quantified using the GeneQuant pro Amersham Biosystem. For the HTLV-1 subtyping, we used a nested-PCR technique, specific for the LTR $5^{\prime}$ region of the HTLV-1, using on the first round, the following primers: ATLTR1 (5'-TGACACTGACCATGAGCCCCAAAT-3') and ATLTR2 (5' TCGTATCCCGGACGAGCCCCCAA $3^{\prime}$ ); and for the second round the primers ATLTR11 (5'-ACTAAGGCTCTGACGTCTCCCCC-3') and ATLTR12 (5'-CGGTACTTGGCCGTGGGCCAAGCCG-3'). For the HIV-1 subtyping, we amplified the envelope (env) and group antigen (gag) regions by nested-PCR. For the env region, during the first round, we used the primers ED5 (5'-ATGGGATCAAGCCTAAAGCCATGTG-3') and MM1 (5'-GGTGAATATCCCTGCCTAA-3'); and in the second round, the primers ED31 (5'-CCTCAGCCATTACACAGGCCTGTCCAAAG-3') and MM4 (5'-CCTCCTACTAT-
CATTATGAA- $3^{\prime}$ ). For the gag region, we used during the first round, the primers GAG1 (5'-GCGAGAGCGTCAGTATTAAGC- $3^{\prime}$ ) and H1P202 (5'-CTAATACTGTATCATCTGCTCCTGT-3'); and in the second round, the primers GAG2 (5'-GGGAAAAAATTCGGTTAAGGCC-3') and G17 (5'-TCCACATTTCCAACAGCCCTTTTTT-3').

The sequencing was performed using the automated sequencer $\mathrm{ABI} 3100$, with the Taq FS Dye Terminator Cycle Sequencing Kit (Applied Biosystems), with the HTLV1 primers: ATLTR11 and ATLTR12; and the HIV-1 primers: ED31, MM4, ES7 (5'-CTGTTAAATGGCAGTCTAGC-3') and ED14 (5'-TCTTGCCTGGAGCTGTTTGATGCCCCAGAC-3') for env; and GAG2, G17, H1G77 (5'-TCACCTAGAACTTTGAATGCATGGG-3') and MZ14 (5' GAACCGGTCTACATAATCTC $3^{\prime}$ ) for gag. To determine subtype, we used the LASP HTLV-1 Automated Subtyping (Version 1.0) tool,

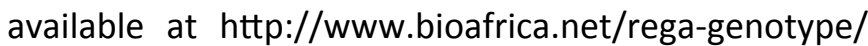
html/subtypinghtlv.html. To determine the HIV subtypes, we used the REGA HIV-1\&2 Automated Subtyping Tool (Version 2.0), available at http://www.bioafrica.net/rega-genotype/html/subtypinghiv.html, which also has tools for the detection of recombinant forms using the Simplot algorithm.

\section{Ethical considerations}

This study was approved by the Research Ethics Committee of Escola Bahiana de Medicina e Saúde Publica under registration number 110/2010. Informed consent was obtained from all participants, authorizing the use of their samples for further studies. In case of minors, consent was signed by parents or guardians.

\section{Results}

A total of 943 pregnant women were enrolled in this study, in which 336 were from llhéus and 647 were from Itabuna Participant's age ranged from 12 to 47 years (average of 24.3 years). The number of pregnancies per women ranged from 1 to 12 (with an average of 2.2), and most women $(87.2 \%)$ reported they never have had history of miscarriage.

Of the total surveyed, 61 pregnant women had a positive serology using filter paper as collecting model, representing an overall prevalence of $6.47 \%$ (61/943), being $8.0 \%(27 / 336)$ in the city of Ilhéus, and $5.6 \%$ $(34 / 607)$ in Itabuna. No cases of coinfection were observed. The specific prevalence for each pathogen was as follows: $0.1 \%$ (1/943) for HCV, $0.2 \%$ (2/943) for HBV, $0.5 \%$ (5/943) for HIV, $1.4 \%$ (13/943) for HTLV, $1.5 \%$ (14/943) IgM positive for $T$. gondii, and 2.8\% (26/943) VDRL positive (T. pallidum).

Of the 61 positive pregnant women in the screening, only $29(47.5 \%)$ returned to collect a venous sample and perform the confirmatory test. One of these had a positive serology for HCV (100\% return - 1/1), two for HIV $(20 \%-2 / 5)$, nine for HTLV $(69 \%-9 / 13)$, nine for T. pallidum (35\% - 9/26) and eight for T. gondii (57\% - 
8/14). Venous blood was collected upon a return visit. Serum from the collection was used to perform an Electrochemiluminescence (EQL) and PCR in case of HIV; ELISA and Western Blot for HTLV; VDRL and FTA-Abs for T. pallidum; IgM search for T. gondii, and anti-HCV search for HCV. After the new tests were performed, we confirmed: the HCV, two samples for HIV and all tested for T. pallidum. There were no false-positives for these three agents. However, of the nine positive samples for HTLV, only six were confirmed $(33.3 \%$ were false-positives) and of the eight returns with positive results for $T$. gondii, only one was confirmed (Table 1).

Upon molecular characterization of retroviruses, the detected HTLV strains in 6 samples clustered within the Cosmopolitan subtype, Transcontinental subgroup (HTLV-1aA) (Table 2). On the other hand, the detected HIV strains in 2 samples clustered with subtypes B and F (F1) (Table 3).

\section{Discussion}

In Brazil, infectious diseases during pregnancy are relatively common, especially affecting disadvantaged populations, and creating challenges to the Public Health system, regarding the establishment of serological screening strategies. On the other hand, studies have revealed that the difficulty encountered by pregnant women to apply for laboratory tests is one of the

Table 1: Analysis of characteristics for 928 pregnant women attended at reference attention service from Southern Bahia State, Brazil, 2009-2010, according to the seropositivity for Rubella, Cytomegalovirus (CMV), Human T lymphotropic virus (HTLV) and Toxopoasma gondii IgG antibodies.

\begin{tabular}{|l|l|l|}
\hline VARIABLE & $\mathbf{N}^{\star}$ & $\%$ \\
\hline Age (years) & & \\
\hline$<20$ & 240 & 25.6 \\
\hline $20-29$ & 499 & 55.0 \\
\hline $30-39$ & 172 & 17.6 \\
\hline$\geq 40$ & 17 & 1.8 \\
\hline Abortion history & & \\
\hline Yes & 100 & 12.8 \\
\hline No & 703 & 87.2 \\
\hline Pregnancies history & & \\
\hline 1 & 335 & 41.1 \\
\hline$\geq 2$ & 499 & 58.9 \\
\hline
\end{tabular}

*Counts might not equal to sample size due to missing data. most significant deficiencies in the quality of prenatal care [21]. It is also important to emphasize that women with multiple pregnancies could have a previous unknown serological status, with a higher possibility of vertical transmission to their children, and underestimating the prevalence of vertical infections.

A study conducted during 2000-2001 in Salvador (capital of Bahia) revealed that $30 \%$ of the 486 surveyed pregnant women did not undergo prenatal care, and most of them attended prenatal care after the second trimester, which reveal a failure in the service [17]. Additionally, the lack of a better effective and integrated network service among healthcare centers, laboratories, and hospitals to provide the results of vertically transmitted infections was another important concern raised in that study [15]. Another study found that only $35.4 \%$ of pregnant women enrolled in the Program for the Humanization of Prenatal and Birth (PHPN) in Brazil has been submitted to HIV testing [16]. According to Cavalcante and collaborators, one of the main barriers to reduce the rates of mother-to-child transmission of HIV, especially in low-income regions, is the complexity of operating a more standardized prenatal care, including the sampling methods and the performance of laboratory tests [18].

In this study, the average number of days to obtain the results of pregnant women was around 20 days. However, the healthcare professionals at the units reported that results for laboratory tests performed by traditional methods took up to 70 days to be available, making difficult any intervention strategies related to positive cases. Although the application of filter paper has already demonstrated to be an efficient method compared to traditional plasma and serum samples collection (including a reduction for the results of routine laboratory tests), we found that the lack of awareness and education of these pregnant women on the importance of prenatal care.

The filter paper methodology has showed a good level of accuracy compared to venipuncture, helping to reduce the waiting time for test results [15]. The sample collection on filter paper also represents a low-cost tool, with a possibility of higher operational capacity, and low-risks during sample transportation and storage, being a feasible method for public services in areas far from major centers and low-resources [14-19]. It

Table 2: Genotyping of HIV sequences using HIV-1 \& REGA 2 Automated subtyping Tool (Version 2.0).

\begin{tabular}{|l|l|l|l|l|l|}
\hline Sample & String length $(\mathrm{bp})$ & Genomic region & Subtype & Bootstrap \%* & Support group for BootScan** \\
\hline TCJ-18 & 956 & Env & - & - & 0.523 \\
\hline TCJ-18 & 954 & Gag & F(F1) & 100 & 1.000 \\
\hline JAL-25 & 1196 & Env & B & 100 & 0.983 \\
\hline JAL-25 & 1016 & Gag & - & - & 0.702 \\
\hline
\end{tabular}

*Only bootstrap values above $70 \%$ were considered significant and described.

"Value given by analysis using the boot scan, where the above 0.900 figures are considered significant. 
Table 3: Subtyping of HTLV-1 LTR region LASP using the HTLV-1 Automated subtyping Tool (Version 1.0).

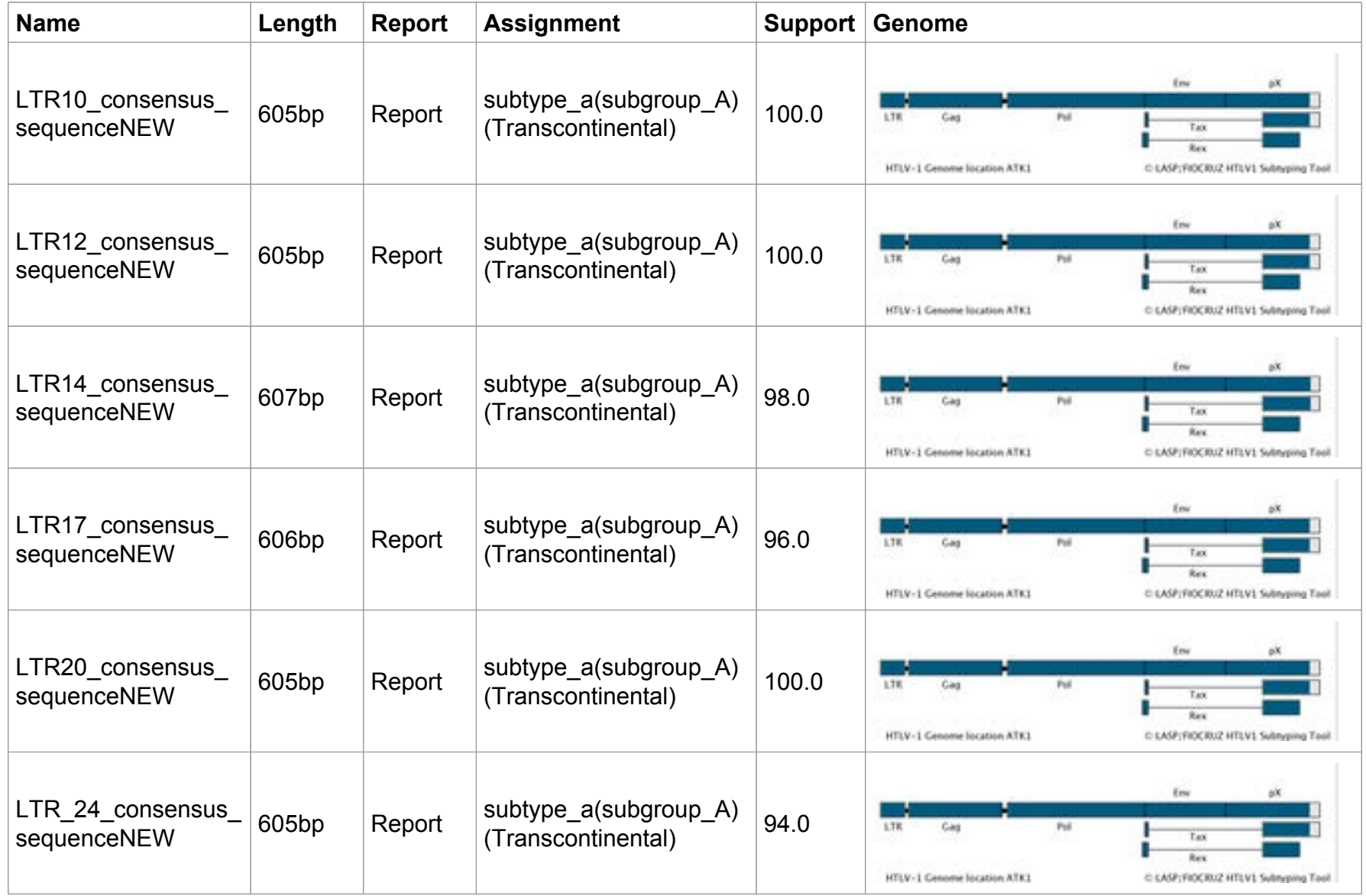

*Only bootstrap values above $70 \%$ were considered significant and described.

can be, therefore, applied as tool for improving prenatal care in the National Health System (SUS) in cities such as Ilhéus and Itabuna, enabling an increase in the number of screened infectious agents, greater accessibility, and reduced time to complete the requested laboratory tests for confirmation. It's also important to say that we do not have access to history of previous laboratory because most women (especially those in low-resource areas) do not follow the Ministry of Health regulations, being the main reasons for not attending prenatal care the lack of properly health education, followed by very low resources or access to the services.

Our study has limitations. Most of women reported they no longer reside in the State, and others refused to return to the Basic Healthcare Units for clinical follow-up, therefore we were unable to obtain detailed clinical information of all patients and unable to collect additional clinical samples. We also found a high amount of false-positive results during screening by using the filter paper technique, especially for HTLV and $T$. gondii, which needs to be further investigated. However, the occurrence of false-positive results is only related to the screening tests, and this data does not compromise the advantages of using the filter paper to ensure a greater inclusion of pregnant women.

\section{Conclusion}

In conclusion, we found important pathogens that can be vertically transmitted in pregnant women from South region of Bahia. We also observed that healthcare professionals and patients in the SUS prenatal service from that region face many difficulties, being the lack of knowledge of vertical transmission pointed as one of the main concerns, reinforcing the need for more incisive health education policies. Although we used tests that promote a fast diagnostic, the rate of return for confirmatory tests was still low, highlighting how the awareness of pre-natal care remains a challenge for the population. Finally, given the severity of the disorders associated to the screened pathogens in this study, checking for the presence or susceptibility to some of these infections needs to be reinforced, mainly for women living in low-resource areas with limited access to healthcare system.

\section{Competing Interests}

The authors declare no conflict of interests in this study.

\section{Funding}

Coordenação de Aperfeicoamento de Pessoal de Nível Superior (CAPES) and Fundação de Amparo ao Pesquisador do Estado da Bahia (FAPESB).

\section{Acknowledgements}

We would like to thank all the professionals from the Primary Care of SUS healthcare facilities in Ilhéus and 
Itabuna cities, Bahia State. We also thank Filipe Almeida Rego for the support with molecular analysis.

\section{References}

1. Jamieson DJ, Theiler RN, Rasmussen SA (2006) Emerging infections and pregnancy. Emerg Infect Dis 12: 1638- 1643.

2. Sappenfield E, Jamieson DJ, Kourtis AP (2013) Pregnancy and susceptibility to infectious diseases. Infect Obstet Gynecol 2013: 752852.

3. Kourtis AP, Read JS, Jamieson DJ (2014) Pregnancy and infection. New Engl J Med 370: 2211-2218.

4. Waldorf KMA, McAdams RM (2013) Influence of infection during pregnancy on fetal development. Reproduction 146 : $151-162$.

5. Suárez-Varela MM, Karlev L, Zhu JL, Llopis-González A Gimeno-Clemente N, et al. (2010) Risk of infection and adverse outcomes among pregnant working women in selected occupational groups: A study in the danish national birth cohort. Environ Health 9: 70.

6. Racicot K, Kwon JY, Aldo P, Silasi M, Mor G (2014) Understanding the complexity of the immune system during pregnancy. Am J Reprod Immunol 72: 107-116.

7. Prabhu DM, Bonney E, Caron K, Dey S, Erlebacher A, et al (2015) Immune mechanisms at the maternal-fetal interface: perspectives and challenges. Nat Immunol 16: 328-334.

8. Arora N, Sadovsky Y, Dermody TS, Coyne CB (2017) Microbial vertical transmission during human pregnancy. Cell Host Microbe 21: 561-567.

9. Costa GB, Oliveira MC, Gadelha SR, Albuquerque GR, Teixeira M, et al. (2018) Infectious diseases during pregnancy in brazil: Seroprevalence and risk factors. J Infect Develp Countries 12: 657-665.

10. Banke-Thomas A, Abejirinde IO, Banke-Thomas $O$, Maikano A, Ameh CA (2019) Cost of maternal health services in low and middle-income countries: Protocol for a systematic review. BMJ Open 9: e027822.

11. Figueiró-Filho EA, Lopes AHA, Senefonte FRA, de Souza Júnior VG, Botelho CA, et al. (2005) T-cell leukemia virus infection in pregnant women in a Central-Western state of Brazil. Rev Bras Ginecol Obstet 27: 719-725.

12. Souza LA, Lopes IG, Maia EL, Azevedo VN, Machado LF, et al. (2006) Molecular characterization of HTLV-1 among patients with tropical spastic paraparesis/HTLV-1 associated myelopathy in Belém, Pará. Rev Soc Bras Med Trop 39: 504-506.

13. Varela MM, Nohr EA, Llopis-González A, Andersen AM, Olsen J (2009) Socio-occupational status and congenital anomalies. Eur J Public Health 19: 161-167.

14. John-Stewart G, Peelning RW, Levin C, Garcia PJ, Mabey D, et al. (2017) Chapter 6: Prevention of Mother-to-Child Transmission of HIV and Syphilis. In: Holmes KK, Bertozzi $\mathrm{S}$, Bloom BR, editors. Major Infectious Diseases. ( $3^{\text {rd }}$ edn), Washington DC, 113-136.

15. Lima BCG, Pimentel CO (2003) Quality of pregnant prenatal care in a public maternity in Salvador, 2000-2001. Rev Baiana Saúde Pública 27: 50-59.

16. Serruya SJ, Cecatti JG, Lago TD (2004) The Brazilian ministry of health's program for humanization of prenatal and childbirth care: Preliminary results. Cad Saúde Pública 20: 1281-1289.

17. Farias JPQ, Franco A, Santos KP, Dourado I, Galvão-Castro B (2008) Prevention of HIV vertical transmission: Obstetricians' attitude in Salvador, Brazil. Rev Bras Ginecol Obstet 30: 135-141.

18. Cavalcante MS, Ramos Júnior AV, Silva TMJ, Pontes LRSK (2004) Human immunodeficiency virus transmission from mother to infant in fortaleza: Revealing the epidemiological situation in a capital from of the Brazilian northeast. Rev Bras Ginecol Obstet 26: 131-138.

19. Snijdewind IJM, van Kampen JJ, Fraaij PL, van der Ende ME, Osterhaus AD, et al. (2012) Current and future applications of dried blood spots in viral disease management. Antiviral Res 93: 309-321.

20. Boa-Sorte N, Purificação A, Amorim T, Assunção L, Reis A, et al. (2014) Dried blood spot testing for the antenatal sreening of HTLV, HIV, syphilis, toxoplasmosis and hepatitis $B$ and C: Prevalence, accuracy and operational aspects. Braz J Infect Dis 18: 618-624.

21. Madhi SA, Rivera LM, Sáez-Llorens X, Menéndez C, Carrim-Ganey N, et al. (2018) Factors influencing access of pregnant women and their infants to their local healthcare system: a prospective, multi-centre, observational study. BMC Pregnancy Childbirth 18: 29. 